\title{
Istanze identitarie e racconto di sé in un gruppo di adolescenti «writers»
}

\section{Barbara De Angelis - Susanna Pallini - Francesca Passarini}

Università degli Studi "Roma Tre», Dipartimento di Scienze della Formazione

doi: 10.7358/ecps-2013-008-dean

barbara.deangelis@uniroma3.it susanna.pallini@uniroma3.it

passarinifrancesca@gmail.com

\section{IDENTITY DEMANDS AND SELF-REPORTS IN A GROUP OF ADOLESCENT GRAFFITI WRITERS}

\begin{abstract}
This paper attempts to investigate the world of graffiti art as a widely popular phenomenon among adolescents, especially in urban contexts. We hypothesize that this activity is an answer to the need for identity and for belonging, typical of adolescence. In particular, we analyzed social identity in the crew and in the broader social context. Twenty young people (aged thirteen to twenty-three years), with nineteen males and only one female, underwent a semi-structured interview on social identity. The transcript was then analysed according to the following categories: the writer's identity (autobiographical, behavioural and emotional aspects along with personal commitment); group identity (the crew, the rules); contextual aspects (belonging, communication goals, relations with the law). The results show two aspects of the activity: on the one hand, graffiti represents an answer to identity needs through its obvious visibility, a revelation and recognition of the self through tagging; on the other hand, it meets the need to belong through the crew's mutual loyalty, the challenge and sharing milieu.
\end{abstract}

Keywords: Adolescence, Identity, Semi-structured interview, Social context, Writers. 


\section{ISTANZE IDENTITARIE E RACCONTO DI SÉ IN UN GRUPPO DI ADOLESCENTI «WRITERS»}

Il termine writer in italiano indica colui che disegna sui muri in specifici posti pubblici utilizzando bombolette di colore acrilico spray, in modo legale o illegale. In realtà il termine inglese esatto è graffiti writer, poiché writer in inglese, significa genericamente colui che scrive. Montoya (2002) ha esaminato la natura ambigua dell'arte dei writers nelle capitali europee quali Parigi, Londra, Amsterdam, Barcellona, Madrid, e negli USA New York. L'autore afferma che attraverso tale pratica, in modo un po' paradossale, i giovani mirano sia a sfidare la società, che a cercare in essa una possibile integrazione. Analogamente Bloch (2002) considera peculiare che atti associabili al vandalismo e al disordine sociale rappresentino un tentativo di porre il proprio segno nel contesto sociale stesso.

Lo spazio urbano diviene un'arena in cui agire una pratica ritualistica e gareggiare per uno status e un riconoscimento (de Oliveira \& Marnoto, 2009), in tal senso possono essere applicate a tale fenomeno vari livelli di lettura: l'arte, la devianza, la politica, la commercializzazione. Non si può prescindere dai risvolti artistici dell'attività di writing in quanto esso rappresenta un'espressione estetica. Ricordiamo, d'altronde, come grandi artisti dell'attualità, quali ad esempio Keith Haring, abbiano cominciato ad esprimersi artisticamente attraverso i graffiti.

La politica è un'altra categoria di lettura, ricordiamo come i graffiti sono stati utilizzati in più contesti nelle lotte di liberazione e in tali casi rappresentavano un messaggio di resistenza o di liberazione. Infine i graffiti possono diventare oggetto di mercato, in quanto possono assumere un valore economico; a partire dagli anni ottanta i media stranieri e il mercato internazionale, hanno prodotto diverse pellicole dedicate al tema (Wild Style, Beat Street, Style Wars) alcune delle quali riproposte nel corso dei festival cinematografici di tutto il mondo (Mininno, 2008); in Italia, invece, sempre più spesso, giovani e affermati writers vengono invitati dietro compenso da aziende ed organizzazioni per realizzare la scenografia o la grafica di prodotti, eventi, manifestazioni, video musicali (è il caso di nominare la Swatch, che nel 2009 ha prodotto la collezione di orologi CreArt in collaborazione con alcuni street artists).

Un'altra importante categoria di lettura è rappresentata dall'aspetto legalitario. Non sempre i writers agiscono in modo illegale, ma a volte o spesso aderiscono ad iniziative gestite o sponsorizzate nella piena legalità. Nonostante ciò, i writers ritengono che se si limitassero ad aderire ad iniziative legali e ad utilizzare spazi loro offerti dalle pubbliche istituzioni, essi perderebbero la loro peculiarità e il prodotto del loro lavoro non sarebbe più arte 
ma mercanzia (Brighenti, 2010). Mentre per altre problematiche adolescenziali è facile applicare delle categorie concettuali dicotomiche quali giusto/ sbagliato, patologico/sano, legale/illegale, per ciò che riguarda il fenomeno del writing opera una commistione di motivazioni e di espressioni (Halsey \& Young, 2006). Alcune caratteristiche accomunano il writing ad altre pratiche giovanili, quali ad esempio lo skateboarding (Borden, 2001; Brighenti, 2010). Ambedue sono attività che si attuano in strada, ma nello stesso tempo sono disseminate in più luoghi e globalizzate. Sono contrastate e combattute dalla legge, ma alimentate dall'idea del valore del desiderio e della creatività. Si basano sull'utilizzo di uno strumento ma nello stesso tempo coinvolgono totalmente il corpo. Possono essere considerate come un retaggio infantile che diviene però uno stile di vita lungo tutto il corso dell'esistenza. Implicano una concezione dello spazio urbano come oggetto non considerato di per sé ma su cui agire per fare esperienza.

Da un punto di vista psicologico il writing può essere accomunato ad altre condotte adolescenziali perché profondamente connesso a due istanze fondamentali in adolescenza: la ricerca dell'identità e il bisogno di appartenenza. Tali istanze sono raccolte da Milio, Scira e Lavanco (2005) nella descrizione dinamica del writing sia come soggettività ed espressione di sé, che desiderio di condivisione e l'appartenenza a qualcosa di più grande, bisogno del gruppo come contenitore psichico e relazionale. Come afferma Wenger (2000), gli aspetti identitari si coniugano alle connessioni che si stabiliscono con gli altri attraverso la condivisione di storie, esperienze ed impegno reciproco.

Per ciò che riguarda il tema dell'identità, secondo Rosci (2003), i writers obbediscono a due istanze adolescenziali collegate al tema dello sviluppo identitario: il bisogno di ammirazione e il tentativo di contrastare la fragilità narcisistica. Per ciò che riguarda il bisogno di ammirazione, la famiglia affettiva di oggi offre molta ammirazione ai figli di tutte le età, ma di una qualità infantilizzante, che rimanda ad un clima relazionale vischioso e dipendente, mentre l'adolescente vuole ammirazione ma senza dipendenza. Il mondo dei writers assicura un senso avvolgente ad una identità virile ancora in formazione. Scrivere in luoghi proibiti diventa una modalità di esorcizzare la paura e di provare la propria virilità e il proprio coraggio. I writers usano le loro attività come strumento per costruire il sé e per la maggior parte il sé maschile.

Come anche affermano Othen-Price (2006) e Macdonald (2001), il nome è al centro di questo processo: il writer non utilizza il suo nome ma ne adotta uno nuovo, collegato ad una nuova identità. In particolare i taggers, che disegnano sugli spazi pubblici soltanto la loro firma o tag sembrano aderire all'idea che «la fragilità narcisistica, il senso della propria pochezza possa essere curato con la notorietà, sinonimo di visibilità» (Rosci, 2003, p. 133). 
L'attenzione alla propria tag diventa un modo per enfatizzare il sé e la propria individualità e nello stesso tempo diviene un modo per accedere alla notorietà e al riconoscimento della comunità più ampia. Di nuovo ci muoviamo dunque tra identità e appartenenza. Secondo MacGillivray e Curwen (2007) un obiettivo del tagging è il desiderio di essere conosciuto o di raggiungere la fama e il riconoscimento in una particolare comunità.

Per ciò che riguarda il tema dell'appartenenza, Halsey e Young (2006) pongono l'accento sulla caratteristica di attività gregaria tipica dei writers, attraverso la quale è facile accedere a un gruppo di amici ben definito. È da tale gruppo di amici, prima ancora che dagli altri, che si vuole essere riconosciuti e apprezzati. Come afferma anche Othen-Price (2006), il writer si pone come oppositore di regole ma nello stesso tempo ritrova all'interno del gruppo (la crew) un altro sistema di regole codificato, rispetto alle quali subisce forti pressioni ad uniformarsi. L'autore assimila il comportamento dei writers all' $\mathrm{i}$ dea eriksoniana (Erikson, 1968) sull'intensa relazione che gli adolescenti stabiliscono con il gruppo, come difesa contro la temporanea perdita d'identità associata con i cambiamenti corporei ed esistenziali propri dell'adolescenza. Gli adolescenti non soltanto si aiutano gli uni con gli altri formando dei comuni stereotipi su loro stessi, i loro ideali e i loro nemici, ma sottopongono se stessi e gli altri a test che provino la loro lealtà negli inevitabili conflitti (Erikson, 1968, p. 133). Le situazioni di emergenza e di pericolo come quelle che si trovano ad affrontare nella loro attività, diventano un banco di prova ottimale della loro virilità e nello stesso tempo della loro lealtà al gruppo. Tali temi identitari emergono in modo privilegiato nelle narrazioni autobiografiche, all'interno delle quali le modalità di descrizione di se stessi e degli altri fanno intravedere le categorie di pensiero privilegiate dai parlanti (Antaki \& Widdicombe, 1998; Rappaport, 1998).

Sia in ambito nazionale che internazionale vi è una crescente mole di ricerca sul fenomeno del writing. In tali ricerche data la difficoltà di lavorare su vasti campioni viene utilizzato un metodo squisitamente qualitativo, basato sull'utilizzo di interviste semistrutturate. Ricordiamo in ambito italiano i lavori di Montesarchio, Margherita e Venuleo (2001), Rosci (2003), Milio, Scira e Lavanco (2005), Montesarchio e Venuleo (2008), Pani e Sagliaschi (2009), Brighenti (2010). In ambito anglosassone citiamo ad esempio i lavori di Austin (2002), Craw e collaboratori (2006), Halsey e Young (2006), MacGillivray e Curwen (2007), Othen-Price (2010). Infine Valle e Weiss (2010) hanno condotto una ricerca etnografica con due crews di giovani artisti di graffiti nella città del Nuovo Messico. Nella ricerca da noi proposta ci concentreremo in particolare modo sul tema dell'identità e dell'appartenenza, come due istanze che si integrano nell'attività di writing. 


\section{OBIETTIVI DELla RICERCA}

Il proposito di esplorare il mondo dei writers è nato nel contesto del progetto educativo territoriale «Boomerang», finanziato dal Municipio XVIII secondo le disposizioni della Legge 285 del 1997. Il progetto prevedeva la creazione di un centro di aggregazione giovanile destinato agli adolescenti a rischio dai 14 ai 21 anni nella zona Bastogi di Roma. In tale laboratorio si trattavano temi quali lo studio del colore, dello stile, della luce e della gestualità. L'intento generale è stato l'indagare la realtà dei graffiti come fenomeno diffuso tra i giovani soprattutto nelle realtà urbane, focalizzandosi in particolar modo su come tale pratica giovanile costituisse una risposta al bisogno d'identità e di appartenenza adolescenziale (Giannotta, Ciairano, Bonino, \& Morero, 2007). L'idea di approfondire tale tematica è nata dall'esperienza di tirocinio di una delle autrici all'interno del «Progetto Boomerang». Il laboratorio preso in considerazione, però, non svolgeva nessun ruolo aggregativo tra gli adolescenti identificati come writers. Per tale motivo è sorta l'esigenza di analizzare il fenomeno del writing attraverso le testimonianze dirette dei giovani che effettivamente si identificavano in tale modello artistico.

In particolare abbiamo inteso indagare aspetti individuali, gruppali e contestuali:

- individuali: autobiografici; comportamentali; emotivi; di impegno personale;

- gruppali: la crew e le regole al suo interno;

- contestuali: l'appartenenza e le intenzioni comunicative.

\section{Metodo}

\subsection{Soggetti}

I soggetti coinvolti sono venti ragazzi di età compresa fra i tredici e i ventitré anni, di cui diciannove maschi ed una sola femmina (Tabella 1).

Tabella 1. - Caratteristiche anagrafiche.

\begin{tabular}{cccccc}
\hline & Medie & Superiori & Università & Lavoro & Totale \\
\hline $13-15$ & 4 & 4 & & & 8 \\
$16-18$ & & 9 & 2 & 1 & 9 \\
$19-23$ & & 13 & 2 & 1 & 3 \\
\hline Totale & 4 & & & & 20 \\
\hline
\end{tabular}


Non è stato possibile interpellare un numero equivalente di maschi e di femmine perché le ragazze che si avvicinano al fenomeno del writing, soprattutto in età pre-adolescenziale, sono molto poche. Tra le possibili motivazioni di questa assenza femminile sono da considerare i diversi interessi che caratterizzano le ragazzine di quell'età, i maggiori controlli da parte dei genitori e gli orari più restrittivi di rientro a casa. Questo però non impedisce loro di essere rispettate e di godere dell'ammirazione e del riconoscimento da parte dei writers di sesso opposto.

Tutti i ragazzi, ad eccezione di un elemento che lavora, sono studenti. Per quanto riguarda le scuole frequentate, quattro soggetti frequentano o hanno frequentato il liceo artistico; cinque hanno frequentato gli istituti professionali (tecnico commerciale, tecnico industriale, alberghiero); tre soggetti hanno frequentato i licei classico e scientifico.

\subsection{Strumenti}

È stato utilizzato un approccio qualitativo. Ai soggetti interpellati è stata proposta un'intervista semi-strutturata, elaborata da De Angelis e Passarini. Tale strumento è stato elaborato allo scopo di investigare il costrutto dell' identità sociale dei writers, come categoria privilegiata sia dal punto di vista interpersonale della $\mathrm{crew}$, sia rispetto al contesto sociale più ampio. Tale metodologia ha consentito di raccogliere dati precisi e approfonditi rispetto ad un elenco predefinito di argomenti, consentendo di adeguare le domande ai singoli intervistati, di modificare ogni volta l'ordine di presentazione delle stesse e di modificare la loro formulazione in itinere, nel caso in cui non fossero correttamente comprese dall'intervistato.

\subsection{Procedura}

L'intero gruppo di intervistati, è stato contattato attraverso alcuni forum in rete frequentati da writers, e nei luoghi di ritrovo tipici degli adolescenti: piazze, centri di aggregazione, stazioni ferroviarie. Vista l'evidente difficoltà di entrare in contatto con giovani che spesso preferiscono mantenere l'anonimato per timore di conseguenze legali, in una prima fase, la modalità di contatto ha previsto la pubblicazione on line della richiesta di collaborazione. In seguito, stabilito il contatto, è stato più semplice chiedere ai partecipanti di partecipare, mantenendo l'anonimato, all'interno di una ricerca sul tema del writing. I soggetti che hanno accettato, hanno concordato un appuntamento con uno degli autori per sviluppare l'intervista. 
I luoghi scelti per l'intervista sono stati i bar e i muretti nei pressi dei maggiori punti di aggregazione per i ragazzi; in particolare sono stati privilegiati spazi riservati, liberi da confusione e privi di elementi di distrazione, per non alterare in alcun modo le risposte.

I soggetti, in tutti i casi, sono stati intervistati singolarmente per evitare che, in presenza di altri, potessero influenzarsi e condizionarsi reciprocamente nel dare le risposte. Inoltre, è sempre stato fornito un foglio con le domande-stimolo, in modo da consentire loro di rileggere più volte il testo senza provare disagio, nel caso in cui avessero incontrato difficoltà nella comprensione delle domande, e in modo che il tempo di lettura fosse anche un momento di riflessione sui contenuti da raccontare e spiegare.

Le interviste sono state di durata variabile: dai 45 minuti ad 1 ora e 15 minuti. Durante tutte le interviste si è cercato di creare un clima positivo, amichevole, colloquiale, basato su una comunicazione circolare, libera e spontanea. Per raccogliere i dati è stato utilizzato un registratore digitale; parallelamente sono stati presi degli appunti sulle parole dell'intervistato.

\subsection{Analisi dei dati}

Le interviste sono state poi trascritte ed analizzate nelle seguenti dimensioni:

- Gli aspetti personali: il modo in cui è avvenuto l'avvicinamento al writing; la scelta della tag; l'impegno dedicato; le emozioni provate.

- L'INTENTO COMUNICATIVO TRA «WRITERS» E COMUNITÀ DI APPARTENENZA: le intenzioni comunicative; gli aspetti autobiografici; le reazioni che si spera di suscitare negli altri.

- LA «CREW» E IL SUO VALORE: il valore del gruppo; le regole e loro condivisione; quali regole.

- La LEgalità/ILlegalità: arte o vandalismo; la differenza tra spazi legali ed illegali; i luoghi del writer; gli ostacoli incontrati nell'attività.

\subsection{Risultati}

- L'identità DEL «WRITER». Gli aspetti personali che sono stati indagati intendono delineare i codici e i valori che caratterizzano il contesto delle relazioni in cui il writer è immerso e sottolineano il significato simbolico affettivo che egli investe nella pratica del writing.

Il percorso. I soggetti sono stati distinti in sei writers inesperti (che praticavano il writing da meno di un anno), otto writers abituali (che praticavano tale 
attività da uno a tre anni) e sei writers esperti, che praticavano tale attività da più di tre anni.

Sono state distinte tre tipologie di percorso:

- attraverso la cultura hip hop;

- influenzati da conoscenti;

- autonomamente.

La maggior parte dei soggetti $(13 / 20)$ ha risposto di essere arrivato al writing come conseguenza dell'incontro con la cultura hip hop, facendo emergere quindi un legame rilevante tra i due fenomeni.

\begin{tabular}{|ll|}
\hline Sogg. 20 aa. & [...] La cultura hip hop si è sviluppata in America negli anni Settanta \\
& ed è arrivata in Italia negli Ottanta; ha quattro grandi sottocategorie: \\
& il writing, il breaking (ballo), il djing e il mcing (canto) [...]. Prima mi \\
& sono accostato alla cultura hip hop (nessuno mi ha influenzato), alla \\
& musica in particolare, e di conseguenza sono arrivata al writing che \\
& mi ha sempre affascinato. Questo è il percorso di quasi tutti i writers, \\
& però in realtà non è obbligatorio [...] però diciamo che nella mag- \\
& gior parte dei casi si riconosce la cultura hip hop come propria. \\
& [...] Mi piace la musica hip hop, mi sono abbonato a una rivista che \\
& ne parla e lì ho visto anche che parlava di urban art, sarebbe il writ- \\
& ing, mi sono iniziato ad incuriosire, poi sempre di più, fino a che non \\
& ho iniziato anch'io.
\end{tabular}

Quattro soggetti hanno subito l'influenza diretta di conoscenti, fratelli, cugini e amici stretti. Tre soggetti si sono entusiasmati per tale fenomeno senza alcuna mediazione.

Sogg. 1319 aa.

[...] Stavamo guardando a scuola delle immagini del muro di Berlino, che è praticamente il più grande museo di graffiti a cielo aperto e così sono rimasto affascinato da quel tipo di arte e da quel modo di esprimere le proprie sensazioni.

La scelta della «tag». La tag è un segno, la firma sul muro che viene realizzata per essere vista e per colpire chi la guarda. Non ha lo scopo di essere letta né di essere capita: è un'impronta che ha una forza espressiva perché ha a che fare con l'identità di chi la traccia. La tag infatti sostituisce il nome ed è qualcosa di strettamente personale: è un nome d'arte che acquista valore non tanto o non esclusivamente per il significato, quanto per la forma del segno grafico.

Le risposte hanno fatto emergere tre ordini di motivazioni:

- fattori estetici e sonori di tutte le lettere dell'alfabeto, o dalle sole lettere del proprio nome; 
- il proprio soprannome o diminutivo;

- un legame con una parola o un nome particolari.

Sette intervistati hanno affermato di aver scelto quelle particolari lettere in seguito ad un semplice, ma attento studio, sui fattori estetici e sonori a partire da tutte le lettere dell'alfabeto.

\begin{tabular}{|ll|}
\hline Sogg. 123 aa. & $\begin{array}{l}\text { [...] Mi piacevano quelle lettere una vicina all'altra, ho fatto uno stu- } \\
\text { dio prima di sceglierle (lettering), non c'è un significato preciso, ora } \\
\text { però quelle lettere sono io. }\end{array}$ \\
\hline Sogg. 2019 aa. & $\begin{array}{l}\text { [...] Sapevo bene quali lettere mi venivano meglio e quali meno, così } \\
\text { ho preso le quattro lettere su cui ero più forte e le ho invertite in vari } \\
\text { modi, finché non ho trovato la combinazione giusta per me. }\end{array}$
\end{tabular}

Per altri (6/20 intervistati) la tag coincide con il soprannome attribuito loro da amici, con il proprio diminutivo o con alcune lettere estratte da essi.

Sogg. 518 a .

[...] Era un soprannome che mi davano i miei amici quand'ero più piccolo, io l'ho accorciato prendendo le lettere più belle da rappresentare graficamente. Di solito la tag è corta perché deve essere facile e veloce da realizzare in qualunque situazione; è molto difficile creare bene se la parola è di per sé lunga, per questo si scelgono nomi corti. Si mi riconosco nella cultura hip hop.

Altri ancora (5/20 intervistati) oltre aver scelto per fattori estetici e di suono, si basano sulle lettere che compongono il proprio nome. Anche in questo caso, i ragazzi hanno sottolineato la difficoltà della decisione.

Sogg. $1319 a a$.

[...] C’ho messo un po' per sceglierlo: ho rivoltato le lettere del mio nome come un pedalino, volevo qualcosa che spaccasse a livello sonoro e che fosse anche graficamente bello da vedere. E così dopo parecchie prove è uscito. Non è una cosa semplice, te l'assicuro!

I restanti $(2 / 20$ intervistati) hanno spiegato che la propria tag è stata scelta a partire da una parola o dal nome di una persona importante per loro ed quindi è costituita da alcune lettere appartenenti a tale nome/parola.

Sogg. 1816 ad. [...] Sono alcune lettere di energy, che in inglese sta per energia. Mi piaceva questa parola, mi rappresenta e così l'ho presa e l'ho personalizzata a modo mio.

L'impegno. Ben nove adolescenti affermano di aver investito un impegno totale; cinque affermano di essersi impegnati tanto o tantissimo, un solo sog- 
getto ha quantificato l'impegno con la categoria abbastanza. I ragazzi hanno fatto luce sull'attento lavoro di preparazione di un pezzo.

\begin{tabular}{|c|c|}
\hline Sogg. $220 a a$. & $\begin{array}{l}\text { [...] Moltissimo (impegno), il pezzo è solo il coronamento di un la- } \\
\text { voro molto dettagliato, fatto di studio, di scelte ... nei giorni pre- } \\
\text { cedenti devi creare il tuo pezzo su un foglio e allora lo cancelli mil- } \\
\text { le volte e lo rifai fino a quando non ottieni la forma migliore. [...] } \\
\text { L'impegno è totale }[. . .] \text { noi non siamo improvvisatori che comprano } \\
\text { una bomboletta a caso e vanno allo sbaraglio dove capita. }\end{array}$ \\
\hline Sogg. $1617 a a$. & $\begin{array}{l}\text { [...] Ogni cosa è studiata ed è il frutto di una scelta precisa: il tipo di } \\
\text { tappetto per la bomboletta, la tonalità di colore, il muro, il posto, il } \\
\text { pezzo da fare ... }\end{array}$ \\
\hline
\end{tabular}

Queste affermazioni trovano un effettivo riscontro con quanto riportato sui testi teorici consultati prima di intraprendere l'indagine: la formazione del writer prevede una costante e profonda dedizione, che lo deve spingere ad aggiornarsi, a migliorarsi, a creare un'identità che sia solo propria in un lungo percorso che richiede cura e impegno trasversale. Ogni writer si impegna nella ricerca di uno stile originale, sperimentando tecniche e forme personali, aggiungendo o togliendo elementi alle sue lettere, cercando un riconoscimento anche attraverso i materiali utilizzati. Nella fase preparatoria tale impegno si concretizza nel progettare le lettere su carta, nel preparare gli accostamenti cromatici e nell'abbozzare la struttura del pezzo su un foglietto da piegare e tenere in tasca.

Le emozioni/sensazioni. Nella Tabella 2 vengono illustrate la frequenza con cui vengono citate le singole emozioni. Le risposte, sono state particolarmente ampie, ricche di motivazioni e riflessioni interpersonali ed anche omogenee dal punto di vista dei contenuti esplicitati.

Tabella 2. - Cosa provo mentre realizzo un "pezzo".

\begin{aligned} & \hline 16 Ebbrezza (brividi, eccitazione, sudorazione, battito accelerato) \\ & 15 Protagonismo, pienezza di sé, potenza, invincibilità \\ & 13 Adrenalina, si sentono a mille, carichi \\ & 9 Felicità \\ & 8 Orgoglio, soddisfazione per il pezzo riuscito \\ & 7 Libertà \\ & 5 Consapevolezza e responsabilità \\ & 2 Leggerezza, tranquillità \\ & 2 Paura \\ & 1 Amore, passione \\ & \hline\end{aligned}


Le risposte sono state varie, pronunciate di getto, istintivamente, con enfasi. Tra la gamma delle sensazioni, un posto primario è occupato dal senso di ebbrezza (nominato da 16 soggetti), inteso come presenza di brividi, eccitazione fisica, elevata sudorazione e battito cardiaco accelerato.

\begin{tabular}{|c|c|}
\hline Sogg. $1514 a d$. & [...] Mi batte il cuore a mille, spesso sudo. \\
\hline Sogg. 1714 aa. & [...] C'è l'adrenalina, l'eccitazione, il batticuore. \\
\hline Sogg. 1319 ad. & $\begin{array}{l}\text { [...] Sono il meglio di ... sono in totale fase di estasi, mi sento un } \\
\text { tutt'uno con la bomboletta! sono sicuro di me, fiero e orgoglioso } \\
\text { (specie quando il pezzo riesce bene). Sto bene in tutti i sensi, è una } \\
\text { valvola di sfogo, ma in positivo, mi scarica e al tempo stesso mi rica- } \\
\text { rica. }\end{array}$ \\
\hline Sogg. $1617 a a$. & $\begin{array}{l}\text { [...] Mi sento me stesso, il vero me stesso sono vivo, non che in al- } \\
\text { tri momenti soffra di depressione, sono vivo nel senso che mi sento } \\
\text { colmo di emozioni che vogliono uscire, a volte sono così emozionato } \\
\text { che mi tremano le mani, mi sudano, mi sento libero di esprimermi e } \\
\text { felice di fare quello che mi piace e mi appassiona. }\end{array}$ \\
\hline Sogg. $1920 \mathrm{ad}$. & $\begin{array}{l}\text { [...] Non credo che tu possa capire bene non avendolo mai provato, } \\
\text { sono sensazioni immense, di pienezza, completezza, libertà e poi più } \\
\text { avanti, più cresci come artista, più sono forti e tu ne diventi sempre } \\
\text { più consapevole. Inizi a conoscerle e sai anche controllarle, però non } \\
\text { vuoi, perché la verità è che le ricerchi con tutto te stesso e non vedi } \\
\text { l'ora che arrivi quel preciso momento per provarle. }\end{array}$ \\
\hline
\end{tabular}

- Il CONTESTO PIÙ AMPIO: L'INTENTO COMUNiCATIVO. Lintento comunicativo si muove tra le due grandi istanze identitarie e di appartenenza: spiegarsi a se stessi e spiegarsi agli altri attraverso la creazione di un linguaggio condiviso fatto di colori e lettere (Lucchetti, 1999). Tale "testo" ha un significato simbolico affettivo che rimanda a rappresentazioni culturali della realtà e che consente di far emergere significati veicolati attraverso questa cultura espressiva (Serra, 2007).

Le intenzioni comunicative. I sentimenti, le emozioni e il proprio mondo sono gli intenti comunicativi che si rilevano con maggiore frequenza. Dodici intervistati vedono le emozioni e i sentimenti come aspetti fondanti l'identità, come oggetti prioritari dell'intento comunicativo.

Sogg. 1920 a .

[...] Voglio esprimere un particolare stato d'animo, un particolare sentimento, anche negativo o voglio farmi conoscere e riconoscere dagli altri. Voglio comunque esprimere qualcosa ogni volta che faccio un pezzo, non è mai un fare tanto per passare il tempo o perché non ho altro per la testa in quel momento. 


\begin{tabular}{|ll|}
\hline Sogg. $1015 \mathrm{ad}$. & $\begin{array}{l}\text { [...] A volte voglio comunicare tanto, inserisco anche intorno alla } \\
\text { scritta effettiva delle parole o delle frasi fatte solo con un'unica linea } \\
\text { (non tutte colorate) come ad esempio: happy, tired, sad ... per sotto- } \\
\text { lineare il mio stato d'animo. }\end{array}$ \\
\hline Sogg. $1015 \mathrm{ad}$. & $\begin{array}{l}\text { [...] Il mio è uno sfogo e quindi più che comunicare, voglio liberar- } \\
\text { mi di qualcosa, sentirmi più leggero. }\end{array}$ \\
\hline
\end{tabular}

Per altri, invece, è prioritario dare sfogo al proprio protagonismo o alla necessità di poter essere identificati come autori di qualcosa di oggettivamente bello.

\begin{tabular}{|ll|}
\hline Sogg. 814 ad. & $\begin{array}{l}{[\ldots] \text { Voglio comunicare la mia voglia di esserci e di urlare questa città }} \\
\text { è anche mia! }\end{array}$ \\
\hline Sogg. $1720 a d$. & $\begin{array}{l}{[\ldots] \text { Non so } \ldots \text { boh!!! Non è che voglio dirti qualcosa di preciso, }} \\
\text { però vorrei che piacessero i miei pezzi. }\end{array}$ \\
\hline
\end{tabular}

Aspetti autobiografici. I giovani intervistati ribadiscono l'utilizzo della pratica del writing come racconto di sé. Alla domanda quanto ritenessero vi fosse di autobiografico nella loro opera, dodici intervistati hanno affermato che è tutto autobiografico; sei che i contenuti sono in gran parte autobiografici e due per nulla. Il soggetto 10 afferma che i pezzi riuniti possano costituire la sua storia. Potremmo dire per analogia che essi rappresentano pezzi della sua identità.

\begin{tabular}{|ll|}
\hline Sogg. 1617 aa. & $\begin{array}{l}\text { [...] È il momento in cui mi scarico di tutto quello che ho provato } \\
\text { nella preparazione del pezzo, nella scelta dei dettagli. Se tu metti in- } \\
\text { sieme vicini tutti i pezzi che ho fatto, puoi benissimo ripercorrere la } \\
\text { mia storia, la mia storia di writer e non solo. È come un album fo- } \\
\text { tografico ordinato cronologicamente in cui puoi renderti conto dei } \\
\text { progressi, del carattere, dello stile ... }\end{array}$ \\
\hline Sogg. 518 aa. & $\begin{array}{l}\text { [...] È come se ti permettessi, attraverso il mio pezzo, di conoscermi } \\
\text { un po' meglio, di entrare nel mio universo. Ti accolgo e ti chiedo allo } \\
\text { stesso tempo di accogliermi. }\end{array}$ \\
\hline Sogg. 919 ad. & $\begin{array}{l}\text { [...] Quel pezzo è la mia storia, racconta qualcosa di me, del mio } \\
\text { modo di essere e del mio stato d'animo in quel particolare momento. } \\
\text { Quando mi capita di passare davanti ad un mio pezzo, mi ritornano } \\
\text { in mente le sensazioni che provavo in quel momento, belle o brutte } \\
\text { che fossero. }\end{array}$ \\
\hline
\end{tabular}

Le reazioni sperate negli altri. In tale gioco dinamico fra il bisogno identitario di spiegarsi a se stessi e bisogno di appartenenze insito nello spiegarsi 
agli altri, si muovono le considerazioni dei nostri soggetti a riguardo delle reazioni sperate da parte degli altri writers e del contesto comunitario di appartenenza.

Tredici soggetti hanno illustrato le reazioni sperate negli altri writers, riconosciuti come gli unici e diretti destinatari delle proprie realizzazioni. In questo caso hanno spiegato in maniera unanime di desiderare il riconoscimento, la stima, il rispetto, la considerazione dei propri compagni di esperienza.

Il bisogno adolescenziale di ammirazione viene soddisfatto attraverso il meccanismo dell'esposizione murale, come un metaforico urlare più forte, per imporsi all'attenzione della comunità.

\begin{tabular}{|ll|}
\hline Sogg. 20 19aa. & $\begin{array}{l}\text { [...] Attenzione dagli altri writers, che può tradursi in rispetto, rico- } \\
\text { noscimento, integrazione al gruppo, presa ad esempio, voglia di un } \\
\text { confronto. }\end{array}$ \\
\hline Sogg. $814 a a$. & $\begin{array}{l}\text { [...] Quello che vorrei veramente è che gli altri writers mi riconosces- } \\
\text { sero e mi ammirassero, e magari mi considerassero come uno degno } \\
\text { di rispetto. }\end{array}$ \\
\hline
\end{tabular}

Tra questi, alcuni hanno mostrato un'indifferenza, almeno apparente, verso le opinioni di chi è esterno al writing; indifferenza che, a tratti, si è esplicitata attraverso un atteggiamento di forzato distacco e superiorità.

In questo caso l'appartenenza ricercata è rigorosamente al gruppo e gli altri, la gente, diventano oggetto di sfida e contrapposizione, contrapposta al gruppo, che si rafforza attraverso tali dinamiche oppositive.

\begin{tabular}{|ll|}
\hline Sogg. $1816 \mathrm{aa}$. & $\begin{array}{l}\text { [...] Del resto (della gente) non è che mi importa tanto, anche per- } \\
\text { ché, trovarne uno che non ci considera delinquenti, non è facile ... }\end{array}$ \\
\hline Sogg. $1919 \mathrm{aa}$. & $\begin{array}{l}\text { [...] Prima mi arrabbiavo, ci rimanevo male della cattiva considera- } \\
\text { zione della gente, ora sinceramente la cosa mi scivola addosso e in al- } \\
\text { cuni casi posso anche capirli. Per questo la cosa più grande che vorrei } \\
\text { è il semplice rispetto, il vedere la gente dire mi fa schifo però va bene, } \\
\text { non mi può piacere per forza sarebbe il massimo! }\end{array}$ \\
\hline
\end{tabular}

Quindici intervistati si sono pronunciati sul rapporto con il contesto comunitario e, quindi, con la gente comune, e sulla volontà di mediare l'attrito che viene percepito come limitante e pesante sotto più punti di vista.

Questo rapporto è comunque descritto come ambivalente e contraddittorio. Le reazioni della gente sono fortemente ricercate, volute da quei writers che sperano in un contatto, in un punto di incontro e di confronto. Le parole riportate di seguito sottolineano questa necessità. 


\begin{tabular}{|c|c|}
\hline Sogg. 123 aa. & $\begin{array}{l}\text { [...] Ammirazione, vorrei che la gente si soffermasse a guardare senza } \\
\text { pregiudizi e preconcetti, che mostrasse curiosità e smettesse di consi- } \\
\text { derare il writer come uno sfidante della società, perché non è così, o, } \\
\text { almeno, non è così nella maggior parte dei casi. }\end{array}$ \\
\hline Sogg. 717 aa. & $\begin{array}{l}\text { [...] La cosa più bella che vorrei è che la gente non associasse più il } \\
\text { writing al vandalismo, al degrado, a famiglie disattente e emarginate. } \\
\text { Vorrei che ci si fermasse a guardare un pezzo e che ci si chiedesse cosa } \\
\text { provava quel ragazzo? cosa sentiva mentre disegnava? come ha usato } \\
\text { qui il colore? }\end{array}$ \\
\hline Sogg. $1617 a a$. & $\begin{array}{l}\text { [...] Oggi come oggi, purtroppo, i writers non contano molto sulle } \\
\text { reazioni della gente comune, perché questa tende a farli passare come } \\
\text { dei delinquenti qualsiasi, come dei ladri o peggio ancora ... Per que- } \\
\text { sto di solito si ricerca il riconoscimento e l'apprezzamento degli altri } \\
\text { writers, soprattutto dei grandi writers. Ottenere la loro approvazione } \\
\text { è il massimo per me. Se fosse possibile però mi piacerebbe che la gen- } \\
\text { te riuscisse ad accettarci. Non dico che poi ogni pezzo deve piacergli, } \\
\text { no, per carità, però almeno arrivare a dire no non mi piace perché } \\
\text { è troppo blu, o perché non capisco cosa voleva dire già sarebbe un } \\
\text { passo avanti importantissimo. }\end{array}$ \\
\hline
\end{tabular}

Nel considerare le reazioni sperate della collettività, comunque, i ragazzi hanno nominato principalmente la curiosità e l'attenzione; il riconoscimento artistico e l'ammirazione; l'approvazione; l'accettazione e la comprensione.

- La «CREW». La crew, quindi il gruppo di pari, è considerato, da quasi la totalità degli intervistati, come un punto di riferimento fondamentale e costituisce una risposta forte al bisogno di appartenenza.

Il valore del gruppo. Le risposte sul significato e il valore della crew sono indicative dell'importanza del ruolo che ricopre il gruppo nell'esperienza del writer e nel suo percorso di costruzione identitaria. Tutti i soggetti, ad eccezione di uno, hanno risposto di far parte di un gruppo, di una crew, anche se alcuni hanno sottolineato di eseguire pezzi con la crew, ma di dedicare anche del tempo a realizzazioni individuali.

Sogg. 518 a a.

[...] Faccio parte di una crew. Tutti i writers fanno parte di una crew, anche se poi non fanno sempre i pezzi insieme. Ci sono i pezzi del singolo e i pezzi della crew. Ovviamente la crew può aiutare il singolo nella realizzazione del suo pezzo, o comunque è quasi sempre presente sul posto quando il singolo disegna.

La crew è stata descritta, da un numero elevato di soggetti, come gruppo di amici; momento di forte aggregazione; aiuto a livello pratico nella realizza- 
zione di un pezzo; supporto psicologico per il singolo writer; quindi come un conforto, un sostegno emotivo, un contenitore di momenti di sfogo; ma anche come una famiglia, ovvero come un luogo di condivisione, di riconoscimento, di consiglio.

Ad alcuni la crew conferisce un senso di appartenenza, protezione e sicurezza; è un luogo dove predomina il rispetto, la lealtà e la fiducia, e i cui appartenenti sono legati tra loro dalla stessa passione. Inoltre, un numero limitato di soggetti guarda ad essa come ad uno spazio di puro e semplice gioco, come una fonte di grande divertimento.

\begin{tabular}{|c|c|}
\hline Sogg. $1920 \mathrm{ad}$. & $\begin{array}{l}\text { [...] La crew per un writer è la base del suo essere writer; è fondamen- } \\
\text { tale perché ti permette un confronto, uno scontro, un conforto e poi } \\
\text { è amicizia vera. C’è totale fiducia: io non vado a fare un pezzo con } \\
\text { qualcuno di cui penso di non potermi fidare. Devo essere sicuro che, } \\
\text { se incontrassi una difficoltà o un imprevisto, vicino a me ci siano } \\
\text { persone pronte a darmi una mano e non a lasciarmi da solo a fare i } \\
\text { conti con il problema [...] poi io passo con la crew gran parte del mio } \\
\text { tempo libero: sono i miei migliori amici, oltre che gli altri membri } \\
\text { della crew. }\end{array}$ \\
\hline Sogg. $220 a a$. & $\begin{array}{l}\text { [...] La crew è come una band: tante entità singole che poi però la- } \\
\text { vorano insieme e danno vita ad un'entità di gruppo. E importan- } \\
\text { tissima: aiuta a livello pratico, normalmente si è amici, si condivide } \\
\text { la stessa passione, sono un supporto per l'individuo e per la crescita } \\
\text { del gruppo stesso, permette un riconoscimento, un'identificazione } \\
\text { in qualcosa di quotidiano (altrimenti il writing è troppo vasto per } \\
\text { sviluppare una vera e propria appartenenza). }\end{array}$ \\
\hline Sogg. $2019 a a$. & $\begin{array}{l}\text { [...] La crew è una famiglia per il writer, in tutti i sensi, si cresce } \\
\text { insieme, come writers e come persone. Si condivide tutto, dai ma- } \\
\text { teriali, ai problemi, ai sogni, alle confidenze quotidiane [...] è come } \\
\text { una comitiva di amici che non è legata solo da un profondo senti- } \\
\text { mento di amicizia, ma anche da una passione comune a cui si dedica } \\
\text { moltissimo tempo [...] se lungo il percorso non subentra una forte } \\
\text { amicizia la crew non va avanti perché la fiducia, il rispetto, la lealtà e } \\
\text { la fedeltà sono tutte cose che non possono non esserci nella crew. }\end{array}$ \\
\hline
\end{tabular}

Anche se il writing è soggettività, modus vivendi ed espressione di sé, in realtà, da queste parole, sembra che a prevalere sia il senso di appartenenza a qualcosa di più ampio, il bisogno del gruppo inteso come contenitore psichico e relazionale (Milio, Scira, \& Lavanco, 2005). Le spiegazioni degli intervistati fanno emergere una realtà che senza il gruppo sarebbe svuotata del suo significato più profondo e potrebbe non esistere nemmeno. L'unico soggetto che ha affermato di non far parte di una crew, ha comunque dichiarato di essere in cerca di un gruppo. Nell'enunciare i possibili vantaggi offerti dall'aggre- 
gazione in una crew egli ha mostrato di essere ancorato a fattori puramente materiali e pratici.

Sogg. 814 aa.

[...] No, non sto in una crew [...] devo trovarmene una mia, magari di coetanei, ma non ho ancora trovato niente; ancora non ho deciso se è fondamentale per un writer; credo che sia utile quando devi raggiungere dei posti difficili o se hai bisogno che qualcuno controlli mentre dipingi, in modo da non essere sorpreso dalle guardie.

Regole condivise. Diciassette intervistati dichiarano l'esistenza di regole precise all'interno della propria $\mathrm{crew}$; al tempo stesso, però, denunciano l'incapacità di descrivere con altrettanta chiarezza e precisione l'esistenza di regole universali, valide per tutti i writers e per tutte le crews, in quanto sta alla coscienza di ciascuno decidere per sé ed insieme al il proprio gruppo. Tre soggetti dichiarano che non ci sono regole.

Le regole, quindi, esistono, ma ogni crew ha le proprie, che non sono necessariamente uguali e condivise dalle altre crews. Come anche affermano Montesarchio e Venuleo (2008), il writing risulta potente e, al tempo stesso, incontrollabile, perché nessuno impone comportamenti, leggi, modi di fare anche se c'è una linea di massima, non si può dire come si veste un writer, non si possono classificare i suoi pensieri, le sue abitudini o le sue scelte politiche.

Sogg. 1617 aa.

[...] Non ci sono regole scritte valide per tutti, ognuno si gestisce a modo suo secondo quello che si aspetta e vuole per la comunità di writers.

Un ragazzo, in particolare ha espresso la sua necessità di avere regole comuni, non tanto per collocare il writing in una categoria universale, quanto per uniformarlo attraverso valori di riferimento universali. Tre soggetti hanno affermato di non condividere regole, anzi hanno manifestato una certa intolleranza verso il termine.

Sogg. $1114 a d$.

[...] No no. E che stiamo a scuola!!!!! Viva la libertà! Niente regole di nessun tipo.

Quali regole. Le regole rivestono un ruolo fondamentale nella cultura dei writers: sono pre-testi organizzativi spesso non esplicitamente dichiarati o diffusi, ma che rappresentano una sorta di «costituzione non scritta», un'etica il cui rispetto è imprescindibile. Hanno come oggetto principale il rispetto di se stessi, del proprio lavoro e di quello degli altri, la difesa dalla sopraffazione e il mantenimento di un clima di unità e armonia nel gruppo. 
Il rispetto nelle sue molteplici forme (verso la propria crew, verso le altre crews, verso i pezzi) è tra le regole maggiormente citate dagli intervistati (10/20).

Importanti sono, inoltre, tutte quelle regole, normalmente condivise, ma non necessariamente esplicitate in un rapporto di amicizia, quindi la lealtà, la sincerità, l'accettazione, la fiducia; ma sono importanti anche aspetti più strettamente pratici, come non arrivare tardi agli appuntamenti oppure onorare il nome della crew con la realizzazione di pezzi importanti.

Per quanto riguarda i rapporti con gli altri writers, in particolar modo se non appartenenti alla propria crew, gli intervistati si sono espressi dichiarando che non bisogna invadere un territorio già rivendicato da altri writers. Non devono, inoltre, essere coperti i pezzi degli altri e bisogna cercare di evitare il più possibile il contrasto con le altre crews e con le autorità.

\begin{tabular}{|l}
\hline Sogg. 1319 ad. \\
a cercare i muri, mi divertivo a casa sui fogli di carta, o al massimo \\
sulle pareti della mia camera. Poi a scuola a una gara di pittura ho \\
conosciuto altri writers della mia scuola e così dopo un po' abbiamo \\
messo su la crew. Non ci siamo più divisi da allora! Siamo cresciuti \\
insieme sia come amici che come writers. A volte litighiamo, ma pen- \\
so sia normale, poi tutto si risolve. Ci vediamo come crew una volta \\
a settimana, poi però ci sono un sacco di altre volte che usciamo per \\
disegnare o per fare tutt'altro. Insieme ce la spacchiamo! È figo per- \\
ché ognuno di noi ha una particolarità ed è quindi un writer «specia- \\
lizzato in» (Phin ad esempio disegna solo pesciolini colorati) poi però \\
quando facciamo il pezzo della crew mettiamo insieme tutto quello \\
che siamo individualmente, fondiamo le diverse esperienze ed esco- \\
no fuori delle cose meravigliose. Ovviamente poi si discute scher- \\
zosamente sui meriti dell'opera ... Per me la crew è fondamentale, \\
perché da solo sì è bello, spesso anzi ricerco un po' di solitudine, pe- \\
rò non c'è quell'arricchimento e quel sostegno in più che solo i tuoi \\
compagni possono darti. Le regole ci sono eccome, però poi c’è chi fa \\
finta di non conoscerle: le crews non devono pestarsi i piedi a vicenda \\
(se c'è un quartiere che è rivendicato da una crew, cioè che è pieno di \\
scritte di una crew particolare, si devono rispettare quegli spazi), non \\
si devono coprire i pezzi di altri, non si imbrattano monumenti, spazi \\
privati o chiese (anche se su questo poi ci sono opinioni divergenti \\
anche tra gli stessi writers) ... Nella mia crew oltre a tutte queste ci so- \\
no poi tutta una serie di regole collegate al rapporto di amicizia: noi \\
siamo un gruppo in tutti i sensi e quindi per funzionare dobbiamo \\
sostenerci, rispettarci, accettarci, confrontarci ... è importante!
\end{tabular}

- Il CONTESTO PIÙ AMPIO: Legalità/Illegalità. Quest'ultimo aspetto rappresenta l'argomento principale nelle odierne discussioni intorno al writing che coinvolgono la collettività e le istituzioni. Ė, al tempo stesso, l'aspetto su cui le opinioni risultano più contrastanti. 
Sono stati indagati i concetti di arte e vandalismo, la differenza tra spazi legali ed illegali, i luoghi del writer, gli ostacoli incontrati.

Arte o vandalismo? Tale domanda è stata formulata senza un soggetto e un predicato espliciti, allo scopo di suscitare una riflessione, un decentramento da sé e di allargare il proprio punto di vista anche al contesto di riferimento. Le risposte sono suddivise in due ambiti:

- il writing è arte;

- il writing non è né arte, né vandalismo.

La maggior parte dei soggetti (16/20) afferma, con convinzione, che si tratta di arte. Tra i motivi di questa scelta: l'enorme impegno profuso; l'accurato studio dei materiali; degli stili; dei luoghi; la preparazione dei bozzetti su carta; l'esistenza di writers riconosciuti come artisti a livello internazionale (Ganz, 2005).

Sogg. 123 ad.

[...] Arte, nulla è improvvisato (solo alcune forme lo sono e lo sono volutamente perché è uno stile particolare) dietro la realizzazione di un pezzo c'è uno scelta accurata, del materiale, del luogo, c'è la preparazione su carta del pezzo che poi verrà realizzato sul muro. Nulla è a caso! Tutto è frutto di uno studio, molto preciso.

Un numero rivelatore di intervistati (9/20), pur avendo descritto la propria attività come arte, ha ammesso la presenza di una parte di vandalismo.

Sogg. 518 aa.

[...] e poi lo ammetto in alcuni casi c'è anche una dose di vandalismo, di voglia di sfida. Inoltre lo devo sottolineare: quello che scrive io e te 3 metri sopra il cielo o fa svastiche, insulti ... non è un writer, però la gente non lo sa e fa di tutta l'erba un fascio.

La causa di ciò, maggiormente citata, è l'azione dei ragazzini (dodici/tredici anni) che si avvicinano al writing in totale inconsapevolezza, senza conoscerne le regole, come frutto di una moda passeggera o come mezzo per sfidare la società di appartenenza e sentirsi in qualche modo potenti. Un intervistato ha spiegato chiaramente tale concetto.

Sogg. $1319 a a$.

[...] Purtroppo accanto all'arte c'è una buona dose di vandalismo, soprattutto nelle fasce d'età più basse: $\mathrm{i}$ ragazzini pensano spesso che il writing sia un modo per sfidare il mondo adulto, per imporsi all'attenzione, di non si sa chi poi, per farsi più grandi agli occhi di altri e quindi commettono errori gravi che vengono condannati dagli stessi writers e che contribuiscono a farci etichettare come devianti, criminali ... 
Per i restanti quattro intervistati il writing non è né arte, né vandalismo.

Sogg. 413 aa.

[...] Con la crew di cui faccio parte abbiamo fatto un motto abbiamo detto: non è arte, non è vandalismo ma è uno stile di vita.

Spazi legali ed illegali. Tutti gli intervistati, ad eccezione di uno, con grande prontezza e decisione, senza il minimo dubbio, hanno risposto di preferire gli spazi illegali, come se la domanda fosse alquanto banale e la risposta non potesse essere che quella.

Alla domanda su quale fosse la differenza tra il dipingere in spazi legali e in spazi illegali, abbiamo due tipologie di risposte:

- non è possibile parlare di una differenza tra spazi legali ed illegali;

- esiste una differenza enorme tra spazi legali ed illegali.

Una minoranza (3/20 intervistati) ha affermato che non è possibile parlare di una differenza perché questo vorrebbe dire discutere di due realtà a pari livello, mentre, a loro parere, attualmente la situazione è ben diversa.

Sogg. $2019 a a$.

[...] La differenza!?! non è possibile parlare di differenza tra muri legali e illegali perché questo vorrebbe dire parlare di muri legali come di una possibile e concreta alternativa ai muri illegali, non è così quindi non è proprio possibile dare vita ad un confronto. Di conseguenza non è possibile parlare di differenza tra i due.

I restanti (17/20 intervistati), invece, credono che la differenza esista, e sia notevole; per descrivere gli elementi che la determinano, i soggetti hanno parlato di illegalità come fattore caratterizzante del writing; del lavoro sulla ricerca della fama e della visibilità possibili solo negli spazi illegali; dello scarso numero degli spazi legali presenti sul territorio; del problema di doversi registrare alla polizia per poter usufruire di spazi legali.

\begin{tabular}{|ll|}
\hline Sogg. 20 aa. & $\begin{array}{l}\text { [...] C’è una differenza immensa, perché ormai per come si è svi- } \\
\text { luppato il fenomeno, l'aspetto illegalità ne è una caratteristica fon- } \\
\text { dante. Mette in moto meccanismi di adrenalina, emozione, voglia di } \\
\text { dimostrare la propria competenza e bravura superando anche questo } \\
\text { ostacolo. }\end{array}$ \\
\hline Sogg. $316 a d$. & $\begin{array}{l}\text { [...] Gli spazi legali sono pochissimi, sempre pieni, non ci prendi gu- } \\
\text { sto mentre negli spazi illegali, senti l'adrenalina che sale finché non } \\
\text { finisci la tua cosa. Poi ti devono schedare per lavorare in spazi legali, } \\
\text { inoltre non tutte le istituzioni li conoscono (una volta ero con un } \\
\text { amico in uno spazio legale è arrivata una volante e ci ha caricato lo } \\
\text { stesso). }\end{array}$ \\
\end{tabular}


Inoltre, alcuni (13/20 intervistati), spontaneamente, hanno menzionato l'eventualità, seppure considerata remota, di una disponibilità maggiore e di una migliore organizzazione degli spazi legali. Tra questi, la maggioranza $(10 / 13)$ crede che, in tal caso, le cose potrebbero cambiare e i muri legali sarebbero finalmente utilizzati in modo massiccio dai writers; i restanti (3/13), invece, sono convinti che la situazione non muterebbe, sia perché l'illegalità rimarrebbe comunque un elemento inscindibile dal writing, sia perché vedono l'eventualità come un'utopia, non veramente possibile e concreta.

I luoghi del writer. Questo tema si è dimostrato un argomento centrale nel dibattito tra gli stessi writers, poiché la maggior parte degli intervistati $(16 / 20)$ è convinta della necessità di stabilire regole comuni che disciplinino il comportamento. Per questo i writers mirano a differenziare i muri utilizzabili da quelli che non lo sono (Serra, 2007), quattro intervistati non accettano limitazioni e affermano di dipingere ovunque credano sia necessario nel momento particolare. Nel descrivere gli spazi maggiormente utilizzati, gli intervistati hanno nominato i muri pubblici, abbandonati o isolati, le carrozze dei treni a breve percorrenza, i vagoni della metropolitana, i lungo-linea ferroviari, i ponti, le gallerie; un numero limitato di soggetti ha, inoltre, elencato i cassonetti, gli spazi pubblicitari, le fermate degli autobus e le saracinesche. Tra gli spazi non utilizzati dai writers quelli indicati dal maggior numero di intervistati sono i monumenti, le chiese e le proprietà private (case, cancelli, negozi, portoni ...); una minoranza, ha aggiunto le automobili e ha dichiarato di non realizzare pezzi su treni e metropolitane a causa delle difficoltà di raggiungere i depositi.

\begin{tabular}{|ll|}
\hline Sogg. 2 20 aa. & $\begin{array}{l}\text { [...] Io dipingo sui muri, sui treni ... Preferisco i luoghi con molta } \\
\text { visibilità, in modo che altri writers possano vedere la mia opera. Però } \\
\text { cerco sempre di non invadere gli spazi privati: i muri di case, i monu- } \\
\text { menti, i negozi ... }\end{array}$ \\
\hline Sogg. 717 aa. & $\begin{array}{l}\text { [...] Io dipingo ovunque è possibile farlo senza creare troppi proble- } \\
\text { mi agli altri, quindi come ti ho già raccontato, niente monumenti, } \\
\text { chiese, portoni di palazzi, muri di case ... mentre mi piace da morire } \\
\text { farlo sui lungolinea ferroviari delle stazioni che frequento (così quan- } \\
\text { do posso mi rivedo), sui vagoni, sui muri abbandonati. }\end{array}$ \\
& $\begin{array}{l}\text { [...] Io ora dipingo sui muri (interni ed esterni) dei casolari abban- } \\
\text { donati, sui lungolinea ferroviari, sulle metro (che spettacolo fare il } \\
\text { pezzo di notte di nascosto e poi aspettare la mattina per vedere uscire } \\
\text { il treno con il tuo pezzo!) sulle fermate dell'auto, sugli spazi dei car- } \\
\text { telloni pubblicitari (meglio vedere una mia bella scritta piuttosto che } \\
\text { i manifesti abusivi dei politici, no!?!). }\end{array}$ \\
&
\end{tabular}


Ostacoli incontrati nell'attività. Il possibile intervento delle forze dell'ordine rappresenta il rischio percepito dalla maggior parte degli intervistati $(11 / 20)$. Un numero consistente di soggetti $(9 / 20)$ ha indicato i passanti come un'incognita poco prevedibile, perché essi possono dimostrarsi ostili o amici, possono essere solidali, chiamare la polizia o addirittura intervenire in prima persona. Altrettanti hanno presentato come ostacoli l'emotività del momento, la concentrazione, l'ispirazione, la scomodità e la consistenza dello spazio scelto. Un numero minore, ma pur sempre rilevante (7/20), ha nominato il poco tempo a disposizione che spinge a semplificare le forme e gli stili; la mancanza di luce adatta e i problemi inerenti le bombolette (6/20), come la temperatura della vernice, l'adeguatezza del tappetto ... Altri ostacoli percepiti sono, inoltre, la stanchezza fisica, i ragazzini incoscienti e inconsapevoli che si definiscono writers $(3 / 20)$ e la presenza di telecamere di controllo $(1 / 20)$.

\begin{tabular}{|ll|}
\hline Sogg. 20 ad. & [...] Può succedere che decidi di fare un pezzo, ti organizzi, vai sul po- \\
& sto e poi ti blocchi ... non hai l'ispirazione, come uno scrittore e quin- \\
& di non combini e hai buttato tempo. Inoltre la comodità dello spazio, \\
& se sei in una posizione scomoda il dito (che spinge sulla bomboletta) ti \\
& si stanca prima e devi fare una fatica enorme. Inoltre, la realizzazione \\
& del pezzo è sempre all'insegna della velocità, perché ti possono scopri- \\
& re in ogni momento e quindi devi per forza ottimizzare il tempo. \\
\hline Sogg. $1319 a a$. & {$[\ldots]$ Vediamo ... il caldo e il freddo che fanno impazzire le bombo- } \\
& lette e non sai mai che esce fuori quando spruzzi, la mano che trema, \\
& la mancanza di luce o la scomodità della location, la porosità del mu- \\
& ro, la fretta ... e anche l'umore personale del writer: a volte sei arrab- \\
& biato o troppo eccitato e non riesci davvero a fare quello per cui eri \\
& partito, allora o ritrovi in fretta la concentrazione o è meglio che lasci \\
& perdere sennò rischi solo di imbrattare il muro e basta. Poi ci sono i \\
& principianti o meglio i ragazzini idioti, loro sono un grande proble- \\
& ma! Vedi, oggi chiunque può comprare una bomboletta, e chiunque \\
& può fare tag sui muri ... c’è la convinzione che fare tag o andare a \\
& scarabochiare i muri equivalga a diventare writer, a diventare forte, \\
& potente, senza limiti ... non è così! questi deficienti non fanno che \\
& peggiorare i giudizi della gente, ci rovinano soltanto!
\end{tabular}

\section{CONCLUSIONI}

A conclusione del nostro percorso conoscitivo sembra possibile ribadire che l'attività di writing offre una risposta sostitutiva alle istanze d'identità, di appartenenza e di regolazione emotiva basata, non su un'ampia visualizzazione del futuro, ma sul soddisfacimento immediato. 
Ai temi identitari, al bisogno di ammirazione, il writing offre risposte legate alla visibilità, al riconoscimento e alla notorietà, conquistati non tanto attraverso un lento percorso di evoluzione, quanto attraverso uno strumento immediato, assimilabile a quello pubblicitario. Sugli spazi pubblici si trovano infatti i messaggi pubblicitari e i graffiti. Attraverso entrambi questi strumenti la notorietà e l'ammirazione viene conquistata non tanto attraverso il contenuto del messaggio, ma principalmente attraverso la modalità di trasmissione.

Ai compiti identitari di conoscenza e consapevolezza di sé il writing offre risposte legate ad un'altra prerogativa di tale pratica, quale il racconto di sé. Il mostrare aspetti di sé sembra sostituire la riflessione su di sé. Lavorare a lungo sul proprio nome per renderlo unico ed originale, deformando e destrutturando le lettere, ponendo comunque attenzione che questo rimanga leggibile, ha la valenza simbolica di prendersi cura di sé. Riprendendo il concetto di Rosci (2003) sull'attenzione alla propria tag come modo per enfatizzare il sé e la propria individualità, questa pratica assume una valenza autocurativa del sentimento di sé e dell'autostima, che può essere enfatizzato dall'educatore. Così come l'educatore potrà raccogliere le potenzialità insite nella capacità dei writers di portare avanti un lungo lavoro di preparazione e di riflessione sull'opera.

Ai bisogni di appartenenza il writer risponde in modo immediato e totale con la crew. L'appartenenza al gruppo dei writers è una appartenenza esclusiva che può precludere altre appartenenze che consentano uno sviluppo identitario più complesso e articolato (cfr. Wenger, 2000). Anche il riconoscimento sociale così auspicato dai writers si riduce in alcuni casi alla comunità ristretta degli stessi writers (MacGillivray \& Curwen, 2007). I legami, le amicizie il gruppo, non vengono scelti e consolidati nel tempo, piuttosto la crew si offre come realtà a cui l'adolescente semplicemente aderisce e che si vitalizza nello svolgimento delle attività congiunte. La crew è una risposta al bisogno di lealtà e di fiducia, che non viene conquistata nel tempo e sedimentata nella memoria, ma ottenuta attraverso l'esperienza di solidarietà/ complicità nelle situazioni di rischio (pochissimi, sono i luoghi spray can art dove si può fare writing e taggare legalmente), dove è immediato il banco di prova delle lealtà reciproche. Solidarietà e complicità sono sostenute da comuni alleanze e comuni nemici, e solo famosi streat artist post graffitari come l'inglese Banksy (2005), l'olandese Neck, l'italiano Eron, possono affermare: «Prima ci cercava la polizia, oggi ci cercano i clienti».

Al bisogno di regolazione emozionale, necessario a consolidare il senso di sé, il writer risponde con la ricerca di sensazioni forti, tipica sia nella creazione del graffito che nel rischio insito in tale attività. Nell'analisi da noi compiuta emerge che nei writers il desiderio di portare avanti un progetto duraturo nel tempo, sembra a volte messo in secondo piano rispetto alla ricerca di sensazioni eccitanti e momentanee. Le emozioni che si giocano nei 
writers vagheggiano un sogno, per i più irrealizzabile, di notorietà, e contemplano momenti di profonda eccitazione, provocati non soltanto dall'impegno creativo, ma anche dalla pericolosità della realizzazione dell'opera.

La connessione tra arte e educazione fortemente sostenuta da J. Dewey in Educazione e arte ci parla di come attraverso tale particolare correlazione sia possibile stimolare degli atteggiamenti positivi utili a riconoscersi, comprendere la propria storia, dare significato al contesto e appropriarsi della realtà, per ristrutturarla e per armonizzarla. L'educatore potrà raccogliere la sfida lanciata dai giovani writers se saprà discernere tra istanze oppositive e istanze di narrazione di sé, e saprà sottolineare il significato della pittura murale come strumento di comunicazione semplice e immediata, in grado di trasmettere messaggi forti ed incisivi per superare le barriere imposte dalla gerarchia sociale e consentire una comunicazione non solo orizzontale (all'interno del popolo), ma anche dal basso verso l'alto (Freire, 1995).

\section{APPENDICE \\ Schema intervista}

Quanti anni hai?

Che studi hai fatto o fai?

Qual è la differenza tra graffiti e murales?

Come hai scelto la tua tag?

Quando ti sei avvicinato al writing?

Perché, qual è stato il tuo percorso?

C'è stato qualcuno o un gruppo che ha influenzato la tua decisione?

Dipingi/disegni da solo o fai parte di una crew?

Mi descrivi la crew, che valore ha la crew nell'esperienza di un writer?

$\mathrm{Ci}$ sono delle regole per i membri di una crew?

Dove dipingi/disegni (su treni, sui muri ...)?

Arte o vandalismo?

Qual è per te la differenza tra il dipingere in spazi legali e in spazi illegali?

Quali di questi due spazi preferisci, e perché?

Pensi che se fossero disponibili maggiori spazi legali i ragazzi dipingerebbero meno in maniera illegale?

Quali sono gli ostacoli maggiori che incontri nella realizzazione delle tue opere?

Quanto impegno investi nell'attività del dipingere?

Quali emozioni e sensazioni fisiche provi mentre dipingi?

Quanto c'è di tuo, di autobiografico nelle tue opere?

Che cosa vuoi comunicare con le tue opere?

Quali reazioni vorresti provocare in chi guarda le tue opere? 
Il «Progetto Boomerang» è un progetto nato nel 1999 e non più rinnovato dopo il 2008; era finanziato dal Comune di Roma (Municipio XVIII), secondo le disposizioni della Legge 285 del 1997, e gestito dall'associazione ITCA-FAP ONLUS (Istituto Terziari Cappuccini dell'Addolorata - Formazione Attività Pedagogiche). Oggetto dell'intervento era l'educativa territoriale, intesa come una prospettiva di pianificazione, progettazione e ricerca nell'ambito delle politiche per l'infanzia e l'adolescenza. La sua azione era orientata alla valorizzazione, all'attivazione e alla promozione delle risorse della comunità locale, attraverso la messa in campo di molteplici servizi. L'utenza a cui si rivolgeva sono i giovani dell'area Bastogi di Roma di età compresa tra i 14 e i 21 anni.

\section{RIFERIMENTI BIBLIOGRAFICI}

Antaki, C., \& Widdicombe, S (1998). Identity as an achievement and as a tool. In Identities in talk (pp. 1-14). London: Sage.

Austin, J. (2002). Taking the train: How graffiti art became an urban crisis in New York City. NewYork: Columbia Univesity Press.

Banksy (2005). Wall and piece. London: Random House.

Bloch, A. (2002). Facades of disobedience: Tags, graffiti and wall frescoes. Revue Des Sciences Sociales, 29, 92-99.

Borden, I. (2001). Skateboarding, space and the city: Architecture and the body. New York: Berg.

Brighenti, A. (2010). At the wall: Graffiti writers, urban territoriality, and the public domain. Space and Culture, 13, 315-322.

Craw, J. P., Leland, L. S., Bussell, M. G., Munday, S. J., \& Walsh, K. (2006). The mural as graffiti deterrence. Environment and Behaviour, 38(3), 422-434.

de Oliveira, C., \& Marnoto, R. (2009). «All city» european graffiti as communication andtransgression in urban settings. Revista de Antropologia, 52(2), 11-46.

Dewey, J. (1999). Educazione e arte. Firenze: La Nuova Italia.

Erikson, E. H. (1968). Identity youth and crisis. New York: Norton \& Company Inc. (trad. it. Gioventù e crisi di identità. Roma: Armando, 1974).

Foucault, M. (1978). La volontà di sapere. Trad. it. di P. Pasquino e G. Procacci. Roma: Feltrinelli.

Freire, P., Gadotti, M., \& Guimaraes, S. (1995). Pedagogia: dialogo e conflitto. Torino: SEI.

Ganz, N. (2005). Graffiti world. Street art dai cinque continenti. Milano: L'Ippocampo. 
Giannotta, F., Ciairano, S., Bonino, S., \& Morero, D. (2007). La percezione del rischio negli adolescenti: le fonti di informazione e le possibilità di comunicazione con adulti e coetanei. Psicologia Clinica dello Sviluppo, 11(1).

Halsey, M., \& Young, A. (2006). Our desires are ungovernable: Writing graffiti in urban space. Theoretical Criminology, 10(3), 275-306.

Lucchetti, D. (1999). Writing. Storia, linguaggi, arte nei graffiti di strada. Roma: Castelvecchi.

Macdonald, N. (2001). The graffiti subculture: Youth, masculinity and identity in London and New York. Basingstoke: Palgrove (Retrieved November 3, 2008).

MacGillivray, L., \& Curwen, M. S. (2007). Tagging as a social literacy practice. Journal of Adolescent \& Adult Literacy, 50(5), 354-369.

Milio, A. G., Scira B., \& Lavanco, G. (2005). Writing. Narrazione dei non luoghi urbani. In G. Lavanco \& C. Novara, Marginalia. Psicologia di comunità e ricerche-intervento sul disagio giovanile. Milano: Franco Angeli.

Mininno, A. (2008), Graffiti writing. Origini, significati, tecniche e protagonisti in Italia. Milano: Mondadori Arte.

Montesarchio, G., Margherita, V., \& Venuleo, C. (2001). Gruppi marginali: writers, modelli culturali e trasformazioni narrative. Gruppi, 2, 115-129.

Montesarchio, G., \& Venuleo, C. (2008). I writers: narrazione creativa di un gruppo marginale giovanile. In A. M. Di Santo (a cura di), Paradossi della mente giovanile. Roma: Borla.

Montoya, E. (2002). Hiphop graffiti: A plague of artists. Politica y Sociedad, 39(2), 361-375.

Othen-Price, L. (2006). Making their mark: A psychodynamic view of adolescent graffiti writing. Psychodynamic Practice, 12(1), 5-17.

Pani, R., \& Sagliaschi, S. (2009). Psychopathology of excitatory and compulsive aspects of vandalistic graffiti. Psychological Reports, 105, 1027-1038.

Rappaport, J. (1998). The art of social change: Community narratives as resources for individual and collective identity. In X. B. Arriaga \& S. Oskamp, Addressing community problems. London: Sage.

Rosci, E. (2003). I tagger: firme metropolitane fra trasgressione e espressività. In Id. (a cura di), Fare male, farsi male. Adolescenti che aggrediscono il mondo e se stessi. Milano: Franco Angeli.

Serra, C. (2007). Murales e graffiti. Il linguaggio del disagio e della diversità. Milano: Giuffrè.

Slim (2007). Roma writing: la città e i suoi graffiti. Roma: Form Act.

Valle, I., \& Weiss, E. (2009). Participation in the figured world of graffiti. Theaching and Teacher Education. http://www.sciencedirect.com/science/ journal/0742051X, 128-135.

Wenger, E. (2000). Communities of practice and social learning systems. Organization, $7(2), 225-246$. 


\section{RiassunTO}

Con il presente lavoro ci siamo proposti di indagare la realtà dei graffiti come fenomeno diffuso tra i giovani soprattutto nelle realtà urbane, come risposta al bisogno d'identità e di appartenenza adolescenziale. In particolare abbiamo inteso analizzare l'identità sociale dei partecipanti relativamente alla "crew" e al contesto sociale piu ampio. A venti ragazzi di età compresa tra i tredici e i ventitré anni; di cui diciannove maschi ed una sola femmina, è stata sottoposta unintervista semi-strutturata sullidentità sociale che è stata poi trascritta ed analizzata secondo le seguenti categorie: identità del «writer» (aspetti autobiografici, comportamentali, emotivi e di impegno personale); identità gruppale (la "crew», le regole); identità contestuale (l'appartenenza sociale, le intenzioni comunicative, il rapporto con la legge). I risultati mostrano come nell'attività dei graffiti da un lato si risponda a bisogni identitari attraverso la visibilità, il racconto di sé e la riconoscibilità attraverso la "tag», dall'altro si risponda ai bisogni di appartenenza attraverso l'adesione alla "crew», banco di prova di lealtà reciproche e luogo di condivisione.

Parole chiave: Adolescenza, Contesto sociale, Identità, Intervista semi-strutturata, Writers. 